\title{
Review of molecular subtyping methodologies used to investigate outbreaks due to multidrug-resistant enteric bacterial pathogens in sub-Saharan Africa
}

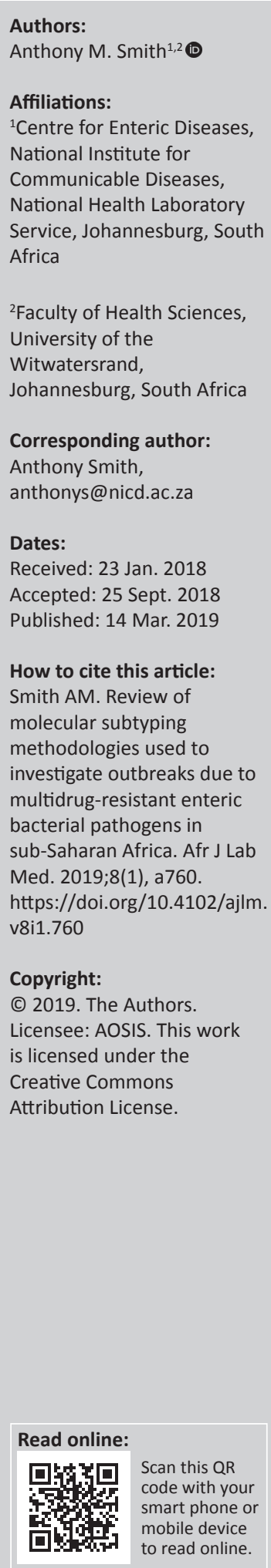

Background: In sub-Saharan Africa, molecular epidemiological investigation of outbreaks caused by antimicrobial-resistant enteric bacterial pathogens have mostly been described for Salmonella species, Vibrio cholerae, Shigella species and Escherichia coli. For these organisms, I reviewed all publications describing the use of molecular subtyping methodologies to investigate outbreaks caused by multidrug-resistant (MDR) enteric bacterial infections.

Objectives: To describe the use of molecular subtyping methodologies to investigate outbreaks caused by MDR enteric bacterial pathogens in sub-Saharan Africa and to describe the current status of molecular subtyping capabilities in the region.

Methods: A PubMed database literature search (English language only) was performed using the search strings: 'Africa outbreak MDR', 'Africa outbreak multi', 'Africa outbreak multidrug', 'Africa outbreak multi drug', 'Africa outbreak resistance', 'Africa outbreak resistant', 'Africa outbreak drug', 'Africa outbreak antibiotic', 'Africa outbreak antimicrobial'. These search strings were used in combination with genus and species names of the organisms listed above. All results were included in the review.

Results: The year 1991 saw one of the first reports describing the use of molecular subtyping methodologies in sub-Saharan Africa; this included the use of plasmid profiling to characterise Salmonella Enteritidis. To date, several methodologies have been used; pulsedfield gel electrophoresis analysis and multilocus sequence typing have been the most commonly used methodologies. Investigations have particularly highlighted the emergence and spread of MDR clones; these include Salmonella Typhi H58 and Salmonella Typhimurium ST313 clones. In recent times, whole-genome sequencing (WGS) analysis approaches have increasingly been used.

Conclusion: Traditional molecular subtyping methodologies are still commonly used and still have their place in investigations; however, WGS approaches have increasingly been used and are slowly gaining a stronghold. African laboratories need to start adapting their molecular surveillance methodologies to include WGS, as it is foreseen that WGS analysis will eventually replace all traditional methodologies.

\section{Introduction}

For molecular epidemiological investigation of outbreaks in sub-Saharan Africa caused by antimicrobial-resistant (including multidrug-resistant [MDR]) enteric bacterial pathogens, most published data describe pathogens belonging to the genus or species of Salmonella, Vibrio cholerae, Shigella and Escherichia coli. By 'molecular epidemiological investigation', I refer to the use of molecular subtyping techniques that analyse bacterial strains at the level of their nucleic acid and so gives an indication of genetic similarity of strains. ${ }^{1}$ Molecular subtyping is vital for accurate epidemiological investigations of bacterial infections. ${ }^{2}$ Molecular subtyping allows one to segregate unlike strains of the same species or serotype of bacteria and identify clones or clusters of bacteria (genetically related strains). Molecular subtyping allows one to track the spread of strains or clones and determine how local strains compare to those circulating worldwide. ${ }^{3}$ Molecular subtyping data is critical for successful epidemiological investigation of outbreaks of disease; in particular, outbreak-related cases of disease can be differentiated from sporadic cases of disease. ${ }^{4}$ Knowledge of the molecular epidemiology of bacterial infections provides information of major circulating (infecting) clones, so that in times of antimicrobial treatment and vaccine interventions, well-informed and educated decisions can be made to combat the disease. ${ }^{5}$ 
The mid-1990s saw the start of noteworthy publications describing the use of molecular subtyping techniques to investigate the molecular epidemiology of antimicrobialresistant enteric bacterial pathogens in sub-Saharan Africa. ${ }^{6}$ These molecular subtyping techniques included plasmid profiling, ribotyping (southern blotting of restricted genomic DNA and probing with ribosomal genes), random amplified polymorphic DNA analysis (also called arbitrarily primed polymerase chain reaction $[\mathrm{PCR}])$, enterobacterial repetitive intergenic consensus elements PCR, pulsed-field gel electrophoresis (PFGE) analysis, multiple-locus variablenumber tandem-repeats analysis (MLVA), multilocus sequence typing (MLST), multilocus sequence analysis and whole-genome sequencing (WGS) analysis. Molecular subtyping data from sub-Saharan Africa is summarised in Table 1 and the countries involved are highlighted in Figure 1. To date, the most popular and well documented molecular subtyping techniques have included PFGE, MLVA, MLST and WGS analysis. Traditional molecular subtyping methodologies, such as PFGE, MLVA and MLST, have their advantages and disadvantages; these have been well reviewed elsewhere on numerous occasions. ${ }^{1,2,7,8,9,10,11}$ Some molecular subtyping techniques (such as ribotyping, random amplified polymorphic DNA analysis and enterobacterial repetitive intergenic consensus elements PCR) may only allow a local comparison of bacterial strains within a single laboratory, because the workings of the methodology are difficult to standardise between laboratories, and so the data that is produced cannot be truly and accurately compared between laboratories. In contrast, a technique such as PFGE analysis, has been standardised by PulseNet International (http:/ / www.pulsenetinternational.org/) and is successfully used for inter-laboratory comparison of subtyping data and global comparison of bacterial strains. ${ }^{4}$

Newer approaches to molecular subtyping involve WGS analysis..12,13,14 Analysis of WGS data for molecular epidemiological purposes can include multiple approaches; however, the more popular methods are whole-genome MLST and single nucleotide polymorphisms (SNP) analysis. ${ }^{15}$ WGS data is electronically portable and can easily be shared between laboratories, allowing for a global inter-laboratory comparison of bacterial strains. Current challenges surrounding WGS include how to standardise the quality of WGS data generated and how to standardise the analysis of WGS data, in order to allow for a successful inter-laboratory comparison of analysed WGS data. PulseNet International has taken on this challenge and published their vision for implementation of WGS for global foodborne disease surveillance and global analysis of enteric pathogens. ${ }^{15}$

The aim of the present manuscript is to review all publications that have described the use of molecular subtyping methodologies to investigate outbreaks due to multidrugresistant enteric bacterial pathogens in sub-Saharan Africa. The review focuses on enteric pathogens belonging to the genus and species of Salmonella, Vibrio cholerae, Shigella and Escherichia coli, because most molecular epidemiological investigations have been described for these microorganisms. The aim of this review is to inform the readers about the current status of molecular subtyping capabilities and activities in sub-Saharan Africa and finally suggest that the way forward for molecular subtyping in African laboratories is the implementation of WGS analysis as soon as possible.

A PubMed database literature search (English language only) was performed using the search strings: 'Africa outbreak MDR', 'Africa outbreak multi', 'Africa outbreak multidrug', 'Africa outbreak multi drug', 'Africa outbreak resistance', 'Africa outbreak resistant', 'Africa outbreak drug', 'Africa outbreak antibiotic', 'Africa outbreak antimicrobial'. These search strings were used in combination with genus and species names of the organisms listed above. All results were included in the review.

\section{Nontyphoidal Salmonella}

For nontyphoidal Salmonella (NTS), molecular epidemiological analysis of Salmonella Typhimurium and Salmonella Enteritidis are most commonly described in sub-Saharan Africa. One of the first reports using molecular subtyping techniques to investigate enteric bacterial pathogens isolated in sub-Saharan Africa was a 1991 study that used plasmid profiling (following restriction endonuclease digestion) to distinguish African isolates of Salmonella Enteritidis from United States (US) isolates. ${ }^{16}$ Molecular subtyping of NTS then progressed to PFGE analysis of MDR Salmonella Typhimurium isolated in Kenya, where 64 isolates were grouped into eight PFGE clusters and were described as multiclonal. ${ }^{17}$ Salmonella Typhimurium accounts for a significant proportion of reported cases of NTS-associated invasive disease in sub-Saharan Africa. NTS-associated invasive disease has developed to become a leading public health challenge in sub-Saharan Africa. ${ }^{18,19}$ A predominant type of invasive Salmonella Typhimurium in sub-Saharan Africa is a MDR strain that has been designated ST313 (based on its MLST profile). Clonal spread of this MDR ST313 strain was first reported from Kenya and Malawi in 2009, with the strain showing resistance to ampicillin, chloramphenicol, sulphamethoxazole, trimethoprim, streptomycin and kanamycin. ${ }^{20}$ In Kenya, a more recent analysis of Salmonella Typhimurium outbreak isolates, using WGS-SNP analysis, identified a single clade of MDR ST313 strains showing resistance to ampicillin, ceftriaxone, chloramphenicol, sulphamethoxazole and trimethoprim; extended-spectrum beta lactamase (ESBL) genes $b l a_{\mathrm{CTX}-\mathrm{M}-15}, b l a_{\mathrm{TEM}-1}$ and $b l a_{\mathrm{OXA}-1}$ were also harboured by the isolates. ${ }^{21}$ The MDR ST313 has further been reported from Malawi, Nigeria, Democratic Republic of the Congo and South Africa. ${ }^{22,23,24}$ Leekitcharoenphon and coworkers $^{22}$ used MLST, PFGE and WGS-SNP analysis to describe a close relationship between MDR ST313 strains from Nigeria and Democratic Republic of the Congo; strains were isolated from invasive (blood) and non-invasive (stool) specimens; all isolates harboured $b \operatorname{la}_{\mathrm{TEM}_{1}}$ cat $A 1, \operatorname{str} A / B$, sul1, and $d f r A 1$ genes coding for resistance to various classes of antimicrobial agents. Keddy and coworkers ${ }^{23}$ used MLST to investigate Salmonella Typhimurium isolates from South Africa and reported that NTS meningitis in South Africa was highly associated with the MDR ST313 strain. 
TABLE 1: Summary of data from publications that have described the use of molecular subtyping methodologies to investigate antimicrobial-resistant enteric bacterial pathogens isolated in sub-Saharan Africa.

\begin{tabular}{|c|c|c|c|c|c|}
\hline $\begin{array}{l}\text { Year of } \\
\text { publication }\end{array}$ & $\begin{array}{l}\text { Type of molecular } \\
\text { subtyping method used }\end{array}$ & $\begin{array}{l}\text { Enteric pathogen } \\
\text { investigated }\end{array}$ & Country of isolation of the pathogen & $\begin{array}{l}\text { Reason for } \\
\text { investigation }\end{array}$ & $\begin{array}{l}\text { Publication } \\
\text { reference }\end{array}$ \\
\hline 1991 & Plasmid profiling & Salmonella Enteritidis & Unknown countries in Africa & Surveillance & 16 \\
\hline 1996 & PFGE, plasmid profiling & Shigella dysenteriae & Kenya & Outbreak & 6 \\
\hline 1996 & Ribotyping & Vibrio cholerae & Guinea-Bissau & Outbreak & 43 \\
\hline 1997 & Ribotyping & Shigella dysenteriae & South Africa & Outbreak & 74 \\
\hline 1997 & ERIC-PCR & Vibrio cholerae & Angola & Outbreak & 44 \\
\hline 1999 & PFGE & Salmonella Typhimurium & Kenya & Surveillance & 17 \\
\hline 2001 & Ribotyping & Vibrio cholerae & Mozambique, South Africa & Outbreak & 46 \\
\hline 2001 & ERIC-PCR & Vibrio cholerae & Mozambique, South Africa & Outbreak & 47 \\
\hline 2003 & PFGE & DEC & Nigeria & Outbreak & 79 \\
\hline 2004 & PFGE & Salmonella Enteritidis & Tanzania & Outbreak & 28 \\
\hline 2004 & PFGE & Salmonella Typhi & Kenya & Outbreak & 32 \\
\hline 2006 & PFGE & Salmonella Isangi & South Africa & Outbreak & 30 \\
\hline 2007 & PFGE & Vibrio cholerae & South Africa & Outbreak & 56 \\
\hline 2008 & RAPD analysis & Vibrio cholerae & Ghana & Outbreak & 50 \\
\hline 2008 & PFGE & Vibrio cholerae & Namibia & Outbreak & 57 \\
\hline 2009 & MLST & Salmonella Typhimurium & Kenya, Malawi & Surveillance & 20 \\
\hline 2009 & PFGE & Shigella boydii & South Africa & Outbreak & 76 \\
\hline 2009 & PFGE, Ribotyping & Vibrio cholerae & Cameroon & Outbreak & 53 \\
\hline 2009 & Ribotyping, RAPD analysis & Vibrio cholerae & Somalia & Outbreak & 54 \\
\hline 2009 & Ribotyping, RAPD analysis & Vibrio cholerae & Ethiopia & Outbreak & 55 \\
\hline 2010 & PFGE & Salmonella Enteritidis & Nigeria & Outbreak & 27 \\
\hline 2010 & PFGE & Salmonella Typhi & South Africa & Surveillance & 33 \\
\hline 2010 & PFGE & Salmonella Typhi & South Africa & Outbreak & 37 \\
\hline 2010 & WGS-SNP analysis & Salmonella Typhi & Kenya & Outbreak & 39 \\
\hline 2011 & PFGE & Salmonella Enteritidis & South Africa & Outbreak & 26 \\
\hline 2011 & PFGE & Salmonella Typhi & South Africa & Outbreak & 4 \\
\hline 2011 & MLSA & Vibrio cholerae & Ghana & Outbreak & 63 \\
\hline 2011 & WGS-SNP analysis & Vibrio cholerae & Several countries & Research & 64 \\
\hline 2012 & PFGE & DEC & South Africa & Surveillance & 80 \\
\hline 2012 & PFGE & Salmonella Typhi & Malawi, Mozambique & Outbreak & 34 \\
\hline 2012 & PFGE & Salmonella Typhi & Uganda & Outbreak & 35 \\
\hline 2012 & WGS-SNP analysis & Salmonella Typhimurium & Malawi & Surveillance & 24 \\
\hline 2013 & PFGE, MLST, WGS-SNP analysis & Salmonella Typhimurium & DRC, Nigeria & Surveillance & 22 \\
\hline 2013 & PFGE & Vibrio cholerae & South Africa & Outbreak & 58 \\
\hline 2013 & WGS-SNP analysis & Vibrio cholerae & Kenya & Outbreak & 65 \\
\hline 2013 & PFGE, MLSA & Vibrio cholerae & Nigeria & Outbreak & 68 \\
\hline 2014 & PFGE & Salmonella Typhi & Uganda & Outbreak & 36 \\
\hline 2014 & PFGE, MLVA, MLST & Salmonella Typhimurium & South Africa & Outbreak & 31 \\
\hline 2014 & PFGE & Vibrio cholerae & Kenya & Outbreak & 69 \\
\hline 2014 & PFGE & Vibrio cholerae & Sierra Leone & Outbreak & 70 \\
\hline 2015 & WGS-SNP analysis & Salmonella Typhi & Several countries & Research & 5 \\
\hline 2015 & WGS-SNP analysis & Salmonella Typhi & Malawi & Surveillance & 40 \\
\hline 2015 & WGS-SNP analysis & Salmonella Typhi & Zambia & Outbreak & 41 \\
\hline 2015 & WGS-SNP analysis & Salmonella Typhimurium & Kenya & Outbreak & 21 \\
\hline 2015 & MLST & Salmonella Typhimurium & South Africa & Surveillance & 23 \\
\hline 2015 & WGS-SNP analysis & Shigella flexneri & Several countries & Research & 77 \\
\hline 2015 & PFGE & Vibrio cholerae & DRC, Guinea, Togo, Côte d'Ivoire, Mozambique & Outbreak & 71 \\
\hline 2015 & MLVA & Vibrio cholerae & DRC, Zambia, Guinea, Togo & Outbreak & 72 \\
\hline 2016 & WGS-SNP analysis & Salmonella Enteritidis & Several countries & Research & 25 \\
\hline 2016 & WGS-SNP analysis & Salmonella Typhi & Nigeria & Surveillance & 42 \\
\hline 2016 & WGS-SNP analysis & Shigella dysenteriae & Several countries & Research & 78 \\
\hline 2016 & WGS-SNP analysis & Vibrio cholerae & Cameroon & Outbreak & 67 \\
\hline 2016 & PFGE, MLVA, MLST & Vibrio cholerae & Ghana & Outbreak & 73 \\
\hline 2017 & MLVA & Salmonella Enteritidis & South Africa & Outbreak & 29 \\
\hline 2017 & MLVA & Salmonella Typhi & South Africa & Surveillance & 38 \\
\hline 2017 & MLVA, WGS-SNP analysis & Vibrio cholerae & Tanzania & Outbreak & 66 \\
\hline
\end{tabular}

DEC, diarrhoeagenic E. coli; ERIC-PCR, enterobacterial repetitive intergenic consensus polymerase chain reaction; MLSA, multilocus sequence analysis; MLST, multilocus sequence typing, MLVA, multiplelocus variable-number tandem-repeats analysis; PFGE, pulsed-field gel electrophoresis; RAPD, random amplified polymorphic DNA; WGS-SNP, whole-genome sequencing single nucleotide polymorphism. 


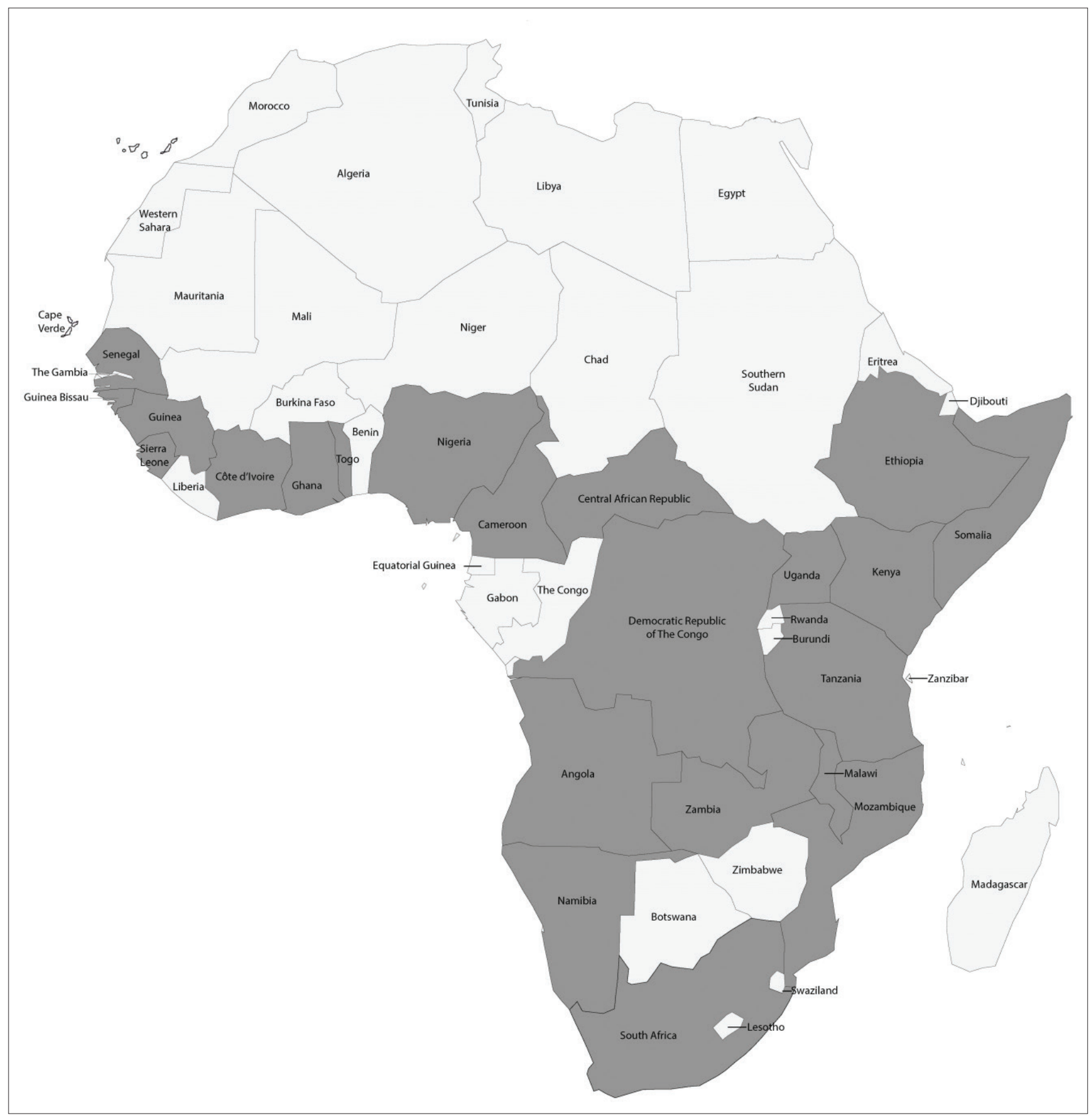

Note: Shaded countries are where resistant pathogens were isolated and further investigated using molecular subtyping methodologies.

FIGURE 1: Map of Africa highlighting (with shading) countries where antimicrobial-resistant enteric bacterial pathogens have been isolated and further investigated using molecular subtyping methodologies.

Besides Salmonella Typhimurium, Salmonella Enteritidis is also a major player with regard to NTS-associated invasive disease in sub-Saharan Africa. Feasey and coworkers ${ }^{25}$ used WGS-SNP analysis to investigate 675 Salmonella Enteritidis isolates from 45 countries (including 28 African countries), to describe the existence of one global epidemic clade and two 'African' clades, of which the African clades are geographically confined to specific regions of sub-Saharan Africa. Both African clades show MDR, with an enlarged MDR virulence plasmid. Both African clades also show patterns of genomic degradation with a similarity to those shown by other host-restricted invasive Salmonella serotypes described in Africa: patterns of genomic degradation similar to the African Salmonella Typhimurium ST313 clade.

Pulsed-field gel electrophoresis analysis is commonly used to investigate outbreaks of Salmonella Enteritidis. ${ }^{26,27,28}$ Niehaus and coworkers ${ }^{26}$ reported on a Salmonella Enteritidis foodborne outbreak in South Africa, where human isolates and a food isolate were shown to have an indistinguishable PFGE pattern. Salmonella Enteritidis investigations have also included nosocomial outbreaks; Vaagland and 
coworkers $^{28}$ investigated a nosocomial outbreak of neonatal Salmonella Enteritidis in Tanzania, with PFGE analysis of MDR (ampicillin, chloramphenicol and cefuroxime) isolates suggesting a clonal outbreak. More recently, MLVA has proven a suitable method to investigate the molecular epidemiology of Salmonella Enteritidis in South Africa; MLVA was used to investigate multiple foodborne outbreaks over the period 2013-2015; MLVA was able to cluster outbreak isolates according to distinct MLVA profiles. ${ }^{29}$ Nosocomial outbreaks have also been investigated in South Africa, where Salmonella Isangi and Salmonella Typhimurium have been involved. ${ }^{30,31}$ Wadula and coworkers ${ }^{30}$ used PFGE analysis to show clonality among ESBL-producing Salmonella Isangi in paediatric hospital wards. Smith and coworkers $^{31}$ used PFGE analysis, MLVA and MLST to investigate an ESBL-producing Salmonella Typhimurium outbreak in a paediatric hospital ward; outbreak isolates showed indistinguishable molecular subtyping profiles including an MLST subtype of ST34, and showed resistance to ampicillin, ceftriaxone, trimethoprim, sulphamethoxazole, chloramphenicol and tetracycline.

\section{Typhoidal Salmonella}

In 2004, Kariuki and coworkers ${ }^{32}$ documented one of the first reports using molecular subtyping techniques to characterise MDR Salmonella Typhi isolated in Africa. They used PFGE analysis to investigate 102 Kenyan outbreak isolates of Salmonella Typhi and identified two distinct subtypes among the infecting isolates; $82 \%$ of the isolates were MDR with resistance to ampicillin, chloramphenicol, tetracycline, streptomycin and cotrimoxazole. When PFGE analysis is standardised, as is the case with PulseNet methodology employed by participating PulseNet International laboratories, then inter-laboratory comparison of PFGE patterns can occur, to allow for a global investigation of outbreak isolates and tracking of emerging strains. Such was the case reported by Smith and coworkers, ${ }^{4}$ who used PFGE analysis to investigate an outbreak of Salmonella Typhi associated with a restaurant in South Africa; the source of the infecting strain was a restaurant worker who was tracked to Australia where a Salmonella Typhi isolate was recovered from the individual; the isolate shared an indistinguishable PFGE pattern as compared to the pattern of the outbreak strain. Using the same scenario as described above (comparison of PFGE patterns within the PulseNet International laboratory network), Keddy and coworkers ${ }^{33}$ investigated a MDR (including resistance to fluoroquinolones) Salmonella Typhi isolate recovered from a South African patient who had interacted with a person who had recently travelled to Bangladesh; the PFGE pattern of the isolate had never previously been seen within the South African PFGE database, but the PFGE pattern was typical of that seen of isolates from the Indian subcontinent, evidence to support the view that the isolate originated in Bangladesh. Global tracking of Salmonella Typhi through inter-laboratory comparison of molecular subtyping data is particularly important in establishing the source (and spread) of MDR (and virulent) strains of Salmonella Typhi. An example of such a strain is Salmonella Typhi H58, a highly clonal MDR haplotype of Salmonella introduced from Asia into Africa and now currently spreading through many sub-Saharan African countries. $^{5}$

Further investigation of Salmonella Typhi outbreaks using PFGE analysis have been reported from the MalawiMozambique border, ${ }^{34}$ in Uganda ${ }^{35,36}$ and in South Africa. ${ }^{37}$ In Uganda, Walters and coworkers ${ }^{36}$ investigated a prolonged waterborne outbreak in two neighbouring districts; they used PFGE analysis to document the clonal spread of MDR Salmonella Typhi from the Kasese district to the Bundibugyo district. Analysis of selected isolates showed MDR to ampicillin, chloramphenicol, cotrimoxazole, streptomycin and tetracycline. In South Africa, Keddy and coworkers ${ }^{37}$ investigated a waterborne outbreak in the Delmas area of South Africa in 2005; they used PFGE analysis and MLVA to show high relatedness among outbreak isolates. These outbreak isolates from 2005 were also shown to be highly related to isolates associated with a similar waterborne outbreak in the same area in 1993. Besides PFGE analysis, MLVA is often described as a useful molecular subtyping method for discrimination of strains belonging to the Salmonella species. Tau and coworkers ${ }^{38}$ described the development and evaluation of a MLVA assay for molecular subtyping of Salmonella Typhi in sub-Saharan Africa. They evaluated the MLVA assay on a panel of African isolates and showed that it had higher discriminatory power as compared to PFGE; the MLVA assay was able to differentiate outbreak isolates from sporadic isolates.

Whole-genome sequencing - single nucleotide polymorphisms analysis has been increasingly used to investigate the molecular epidemiology of Salmonella Typhi in sub-Saharan Africa; a common theme has been the description of the MDR Salmonella Typhi H58 strain. ${ }^{5,39}$ Wong and coworkers ${ }^{5}$ used WGS-SNP analysis to investigate 1832 Salmonella Typhi isolates from 63 global countries, to identify one major MDR lineage (named H58) that has arisen and spread across Asia and Africa over the past 30 years. Multiple H58 transfers have occurred, moving from Asia into Africa. An MDR H58 epidemic has been described in sub-Saharan Africa where H58 lineages are displacing antimicrobial-susceptible strains. MDR H58 lineages are associated with resistance to ampicillin, trimethoprim, sulphonamides, chloramphenicol, streptomycin and tetracycline, including reduced susceptibility to fluoroquinolones. ${ }^{5,39}$ This MDR H58 lineage has now been described in the eastern and southern regions of sub-Saharan Africa including Kenya, Tanzania, Malawi and South Africa. ${ }^{5,38,39,40}$ Hendriksen and coworkers ${ }^{41}$ also used WGS-SNP analysis to investigate an outbreak of Salmonella Typhi in Zambia; most isolates showed resistance to ampicillin, chloramphenicol, streptomycin, sulphamethoxazole and trimethoprim, while some isolates also had reduced susceptibility to fluoroquinolones. Isolates belonged to a new variant of the H58 haplotype, namely haplotype H58B. Interestingly, investigation of MDR 
Salmonella Typhi from Nigeria -western Africa, using WGSSNP analysis, has not detected the H58 lineage; instead the majority of isolates belonged to a different lineage -H56 lineage, which carries genes coding for resistance to ampicillin, tetracycline, chloramphenicol and sulphamethoxazole ( $b l a_{\mathrm{TEM} 1}$, cat $A 1$, tet $B, d f r A 1$, sul1). This H56 lineage is relatively common across Africa, predominantly in western and central regions of sub-Saharan Africa. ${ }^{42}$

\section{Vibrio cholerae}

Ribotyping and enterobacterial repetitive intergenic consensus elements PCR were among the first molecular subtyping techniques used to characterise $V$. cholerae $\mathrm{O} 1$ in Africa. ${ }^{43,44}$ In 1996, Dalsgaard and coworkers ${ }^{43}$ documented the first molecular epidemiological investigations of MDR $V$. cholerae $\mathrm{O} 1$ in Africa. They used ribotyping to investigate outbreak isolates in Guinea-Bissau; strains isolated in 1994-1995 showed a ribotype pattern which was different fro strains isolated in 1987, suggesting that the 1994-1995 outbreak was as a result of the introduction of a novel strain into the country. Later, ribotyping analysis of $V$. cholerae $\mathrm{O} 1$ outbreak isolates from Senegal showed the same ribotype pattern as the 1994-1995 Guinea-Bissau outbreak strain, suggesting that Senegal acquired their outbreak strain from Guinea-Bissau. ${ }^{45}$ In 2001, Dalsgaard and coworkers ${ }^{46}$ reported on the clonal relationship (using ribotyping) of cholera isolates from Mozambican migrant workers associated with a 1998 outbreak that occurred in South African provinces bordering Mozambique. MDR V. cholerae $\mathrm{O} 1$ isolates were associated with the outbreak among the migrant workers and the isolates showed resistance to furazolidone, streptomycin, sulphamethoxazole, trimethoprim and tetracycline; isolates also showed the presence of class 1 integrons and the SXT element. Another analysis of Mozambican MDR isolates associated with the 1998 Mozambique/South Africa cholera outbreak confirmed the presence of the SXT element among isolates, with enterobacterial repetitive intergenic consensus elements PCR dividing isolates into two different molecular subtypes. ${ }^{47}$ Mobile genetic elements are mostly responsible for the molecular basis of MDR $V$. cholerae $\mathrm{O} 1$ in sub-Saharan Africa. Mobile genetic elements include transposable elements (SXT elements), integrons and conjugative plasmids. The SXT element is a self-transmissible element that integrates into the chromosome and carries genes encoding resistance to several antimicrobial agents, including chloramphenicol, sulfamethoxazole, streptomycin, trimethoprim and furazolidone. The SXT element has been reported from numerous countries in sub-Saharan Africa. ${ }^{46,48,49,50,51}$ In 2006, Scrascia and coworkers ${ }^{52}$ investigated $V$. cholerae $\mathrm{O} 1$ isolates from multiple outbreaks that occurred in Kenya during 1998 and 1999. Most isolates showed an identical ribotype profile and similar random amplified polymorphic DNA analysis profile suggesting a clonal origin for the outbreaks; isolates were resistant to chloramphenicol, spectinomycin, streptomycin, sulphamethoxazole and trimethoprim. Over the period 2008-2009, published data from Ghana, Cameroon, Ethiopia and Somalia reported similar trends (as described above) for MDR $V$. cholerae $\mathrm{O} 1$ isolates following analysis using ribotyping and random amplified polymorphic DNA analysis - that of each country reporting a clonal origin for outbreak isolates within their respective country. ${ }^{50,53,54,55}$

From 2007 onwards, trends in molecular subtyping of $V$. cholerae, started shifting to an increased use of PFGE analysis. In 2007, Keddy and coworkers ${ }^{56}$ used PFGE analysis to compare the relatedness of South African V. cholerae O1 isolates from a 2001-2002 epidemic to that of a 1980-1987 epidemic; PFGE analysis showed that isolates from the 19801987 epidemic were distinctly different to isolates from the 2001-2002 outbreak. In 2008, Smith and coworkers ${ }^{57}$ reported on the first cholera outbreak in Namibia over the period 2006-2007; MDR V. cholerae O1 isolates showed an indistinguishable PFGE pattern and showed resistance to streptomycin, sulphamethoxazole and trimethoprim. In 2011, Ismail and coworkers ${ }^{48}$ investigated an outbreak of ESBLproducing MDR $V$. cholerae $\mathrm{O} 1$ in South Africa which occurred over the period May to July 2008; PFGE analysis showed a clonal relationship among isolates, with isolates showing resistance to ampicillin, cotrimoxazole, chloramphenicol, nalidixic acid, tetracycline, kanamycin and streptomycin. The molecular basis of antimicrobial resistance was explained by proving the presence of the SXT element and the $b l a_{\mathrm{TEM}}$ gene encoding TEM-63 $\beta$-lactamase. Later in 2008, a V. cholerae O1 outbreak in Zimbabwe spilled over into South Africa, which triggered a very large outbreak in South Africa over the period November 2008 to April 2009. MDR outbreak isolates showed similar PFGE patterns: isolates showed the presence of the SXT element encoding multidrug resistance; some isolates also showed ESBL activity due to the presence of the $b l a_{\text {TEM }}$ gene encoding TEM-63 $\beta$-lactamase. ${ }^{58}$ In October 2010, the Haiti cholera outbreak started, with involvement of MDR $V$. cholerae O1.59,60 PFGE analysis of Haiti outbreak strains isolated over the period October 2010 to February 2011 showed that a single PFGE profile predominated. This predominant profile was also shown in MDR strains from Cameroon, South Africa, India, Nepal, Pakistan, Afghanistan and Oman ${ }^{61}$; this global comparison of $V$. cholerae O1 PFGE patterns was facilitated via PulseNet International laboratories. Further investigation with WGS-SNP analysis showed that the Haiti outbreak strain was a hybrid-type of El Tor strain encoding a classical-type of cholera toxin and that the outbreak strain was most closely related to strains originating from India and Cameroon. ${ }^{62}$

The Haiti cholera outbreak identified the need for higher resolution molecular subtyping methodologies for comparison of $V$. cholerae $\mathrm{O} 1$ isolates; this sparked the increased use of nucleotide sequencing approaches including WGS, to generate nucleotide sequence data for comparison of isolates. In 2011, Thompson and coworkers ${ }^{63}$ characterised $V$. cholerae $\mathrm{O} 1$ outbreak isolates from Ghana using multilocus sequence analysis of housekeeping genes and identified two major clusters in Ghana. In 2011, Mutreja and coworkers ${ }^{64}$ provided evidence for multiple waves of global transmission (from the Bay of Bengal) within the seventh cholera pandemic, which included multiple waves of 
transmission into sub-Saharan Africa; these data were made possible by analysis of WGS data (WGS-SNP analysis approach) from a global collection of $V$. cholerae $\mathrm{O} 1$ isolates, including African outbreak isolates. In 2013, Kiiru and coworkers, ${ }^{65}$ reported a WGS-SNP analysis on Kenyan $V$. cholerae isolates which included environmental isolates (serogroups $\mathrm{O} 1$ and non-O1) and $V$. cholerae O1 clinical isolates associated with outbreaks; they found that a clade comprising clinical isolates and some environmental isolates mapped back onto wave three of the monophyletic seventh cholera pandemic phylogeny, while some other environmental isolates were phylogenetically very different from the monophyletic seventh pandemic lineage of $V$. cholerae O1. The continued presence of MDR associated STX elements were documented on the genomes of MDR clinical and MDR environmental isolates. Recent isolates of $V$. cholerae $\mathrm{O} 1$ from Tanzania have also been found to map back onto wave three of the monophyletic seventh cholera pandemic phylogeny, as determined by WGS-SNP analysis. ${ }^{66}$ Interestingly, WGS-SNP analysis of MDR $V$. cholerae O1 outbreak isolates recently sourced from Cameroon has found that these Cameroonian isolates do not map onto the phylogeny of isolates from other African countries such as Kenya, Tanzania, Zambia, Zimbabwe and Mozambique; instead, Cameroonian isolates are of their own distinct clonal cluster (clade), forming part of an isolated reservoir of MDR $V$. cholerae $\mathrm{O} 1$ in the Lake Chad basin. ${ }^{67}$

Although WGS approaches for comparison of $V$. cholerae isolates have gained a stronghold in sub-Saharan Africa, the traditional molecular subtyping methodologies of PFGE, MLVA and MLST are still commonly used to investigate the molecular epidemiology of V. cholerae in sub-Saharan Africa, as these technologies still remain more accessible and more affordable for most laboratories ${ }^{6,68,69,70,71,72,73}$. For these recent reports, the central themes remain the same as previous studies. The major conclusions for these studies are summarised as follows. The most commonly reported cholera-causing organism is characterised as V. cholerae $\mathrm{O} 1$ biotype El Tor, the so-called 'atypical' El Tor variant housing genetic determinants coding for a 'classical-type' of cholera toxin. The majority of $V$. cholerae $\mathrm{O} 1$ isolates show the presence of SXT elements encoding a large part of the MDR phenotype. The majority of isolates show MDR. A common profile includes resistance to ampicillin, chloramphenicol, sulphamethoxazole, trimethoprim, spectinomycin and streptomycin, including reduced susceptibility to ciprofloxacin. Molecular subtyping of $V$. cholerae $\mathrm{O} 1$ isolates shows examples of geographical clustering, as already described above in the mention of Cameroonian isolates characteristic of the Lake Chad basin region; Moore and coworkers $^{72}$ found that isolates from western Africa (Togo and Guinea) form a closely related group which is separated from a distinctive cluster associated with the African Great Lakes region (Democratic Republic of the Congo and Zambia). Smith and coworkers ${ }^{71}$ found that a Mozambican cluster of isolates was distinctly different (including a different antimicrobial resistance profile) as compared to isolates from western Africa (Togo, Guinea and Côte d'Ivoire) and central Africa (Democratic Republic of the Congo).

\section{Shigella species}

Shigella dysenteriae was the first Shigella species in sub-Saharan Africa that was characterised by molecular subtyping techniques; these details were first documented in the years 1996 and 1997. ${ }^{6,74}$ In 1996, Kariuki and coworkers ${ }^{6}$ documented an outbreak of dysentery due to MDR S. dysenteriae serotype 1 in Kenya: all 22 outbreak isolates were resistant to ampicillin, chloramphenicol, sulphamethoxazole, trimethoprim, streptomycin and tetracycline; PFGE and plasmid profiling showed clonality among the isolates. In 1997, Pillay and coworkers $^{74}$ documented a nosocomial outbreak of dysentery caused by MDR S. dysenteriae serotype 1 which transpired in a psychiatric institution in South Africa. Isolates were resistant to ampicillin, chloramphenicol, tetracycline and cotrimoxazole; ribotyping showed clonality among the isolates. Later in 1996, a report documented how PFGE analysis was used to investigate MDR S. dysenteriae serotype 1 isolates associated with two separate dysentery outbreaks in the Central African Republic; PFGE analysis provided evidence to show that each outbreak was associated with a different clone. ${ }^{75}$ In 2009, Smith and coworkers $^{76}$ reported a cluster of 29 watery diarrhoea cases associated with MDR Shigella boydii serotype 2 isolates in South Africa. Isolates were resistant to ampicillin, trimethoprim, sulphamethoxazole and streptomycin; PFGE analysis showed clonality among the isolates.

More recent molecular epidemiological investigations of Shigella species in sub-Saharan Africa has involved WGSSNP analysis of isolates. Connor and coworkers ${ }^{77}$ used WGSSNP analysis to investigate 351 global (including Africa) isolates of Shigella flexneri, to show that S. flexneri is composed of seven distinct phylogenetic groups. Each phylogenetic group contains geographically restricted sub-lineages that seem to have constantly inhabited areas for several decades; there is limited evidence of intercontinental transmission. Antimicrobial resistance determinants have been acquired and maintained locally on several episodes - to summarise, S. flexneri from sub-Saharan Africa cluster together and form a distinct lineage as compared to lineages from other parts of the world. Njamkepo and coworkers ${ }^{78}$ used WGS-SNP analysis to investigate 331 global (including Africa) isolates of $S$. dysenteriae serotype 1 , to show that $S$. dysenteriae serotype 1 is composed of four distinct lineages. Lineage IV contains most of the recent (last few decades) isolates from Africa and the Indian subcontinent; lineage IV has been transmitted in several waves to Africa; most recent (since 1990) outbreaks in Africa have been caused by this lineage IV; compared to other lineages, lineage IV show antimicrobial resistance including MDR.

\section{Escherichia coli}

Surprisingly very little published data exist concerning molecular epidemiological investigation of outbreaks in sub-Saharan Africa caused by E. coli enteric 
pathogens (diarrhoeagenic E. coli). Diarrhoeagenic E. coli includes the following six main categories: shiga-toxin producing E. coli (which includes the enterohaemorrhagic E. coli), enteropathogenic E. coli, enteroinvasive E. coli, enterotoxigenic E. coli, enteroaggregative E. coli and diffusely adherent E. coli. In 2003, Okeke and coworkers ${ }^{79}$ reported on the etiology of acute diarrhoea in adults in Nigeria: shiga-toxin producing E. coli and enteroaggregative E. coli were significantly associated with the diarrhoea. PFGE analysis of shiga-toxin producing E. coli isolates provided evidence to suggest that an outbreak had occurred. In 2012, Tau and coworkers $^{80}$ reported on the characterisation of E. coli O104 isolates from South Africa for the years 2004-2011. This study was undertaken in response to the 2011 outbreak of bloody diarrhoea and haemolytic uraemic syndrome which occurred in Germany, caused by an enteroaggregative shiga-toxin producing E. coli O104: $\mathrm{H} 4^{81}$. The South African investigation found two antimicrobial-susceptible enteropathogenic E. coli O104:non-H4 isolates and five enteroaggregative E. coli O104:H4 isolates (showing resistance to ampicillin, sulphamethoxazole and trimethoprim). Pulsed-field gel electrophoresis analysis showed that these South African isolates were unrelated to the Germany outbreak strain.

\section{Conclusion}

Whole-genome sequencing is undoubtedly the way forward for investigation of enteric pathogens in diagnostic and public health laboratories. Numerous publications have analysed data sets to compare results obtained using old-fashioned molecular subtyping methodologies versus results obtained using newer WGS analysis methodologies. ${ }^{14,82,83,84}$ Conclusions from these studies are unanimous and convincing, in that WGS trumps all other methodologies. Old-fashioned methodologies offer limited genetic resolution and limited discriminatory ability and there can sometimes be a lack of accuracy; all these hinder epidemiologic investigations. For example, data from oldfashioned methodologies can incorrectly conclude that unrelated isolates are indistinguishable, resulting in false alerts and unnecessary investigations. Whole-genome sequencing provides a supreme unparalleled discriminatory ability, can reflect phylogeny and can provide an evolutionary context. In addition, WGS can provide valuable information related to genetic determinants conferring antimicrobial resistance. Numerous publications have reported how analysis of WGS data can be used to infer antimicrobial resistance in bacterial pathogens; $85,86,87,88,89$ these studies have reported high concordance between WGS resistance predictions and conventional phenotypic antimicrobial susceptibility testing methodologies. Even so, conventional phenotypic susceptibility testing methodologies still have their place in the laboratory and must continue to be used for the foreseeable future. For now, WGS antimicrobial resistance prediction is best used as an adjunct to conventional phenotypic susceptibility testing.

In summary, public health laboratories in Africa need to urgently reconsider and adapt their molecular surveillance methodologies to include WGS. It is foreseen that WGS analysis will eventually replace all traditional methods currently used in the microbiology laboratory, both traditional phenotypic methods and traditional genotypic methods including molecular subtyping methods such as PFGE, MLVA and MLST.90,91,92,93 Within the next year, the PulseNet USA programme is planning to have WGS (and analysis of WGS data) fully implemented as its primary method for molecular epidemiological investigation of enteric pathogens. ${ }^{94}$ The next challenge is how to effectively implement WGS analysis globally in public health laboratories, how to standardise the quality of WGS data generated and how to standardise the analysis of WGS data, all in order to allow for a successful inter-laboratory comparison of analysed WGS data. PulseNet International has taken this challenge and published their vision for implementation of WGS for global foodborne disease surveillance and global analysis of enteric pathogens. ${ }^{15}$ PulseNet Africa (a regional network of PulseNet International) believes and supports this vision for implementation of WGS for global analysis of enteric pathogens.

\section{Acknowledgements Competing interests}

The author declares that he has no financial or personal relationships that may have inappropriately influenced him in writing this article.

\section{Sources of support}

None.

\section{References}

1. Van Belkum A, Tassios PT, Dijkshoorn L, et al. Guidelines for the validation and application of typing methods for use in bacterial epidemiology. Clin Microbio Infect. 2007;13(Suppl3):1-46. https://doi.org/10.1111/j.1469-0691.2007.01786.x

2. Sabat AJ, Budimir A, Nashev D, et al. Overview of molecular typing methods for outbreak detection and epidemiological surveillance. Euro Surveill. 2013;18(4):20380. https://doi.org/10.2807/ese.18.04.20380-en

3. Swaminathan B, Gerner-Smidt P, Ng LK, et al. Building PulseNet International: An interconnected system of laboratory networks to facilitate timely public health recognition and response to foodborne disease outbreaks and emerging recognition and response to foodborne disease outbreaks and emerging
foodborne diseases. Foodborne Pathog Dis. 2006;3(1):36-50. https://doi. org/10.1089/fpd.2006.3.36

4. Smith $\mathrm{AM}$, Keddy $\mathrm{KH}$, Ismail $\mathrm{H}$, et al. International collaboration tracks typhoid fever cases over two continents from South Africa to Australia. J Med Microbiol. 2011;60:1405-1407. https://doi.org/10.1099/jmm.0.030700-0

5. Wong VK, Baker S, Pickard DJ, et al. Phylogeographical analysis of the dominant multidrug-resistant $\mathrm{H} 58$ clade of Salmonella Typhi identifies inter- and intracontinental transmission events. Nat Genet. 2015;47(6):632-639. https:// doi.org/10.1038/ng.3281

6. Kariuki S, Muthotho N, Kimari J, Waiyaki P, Hart CA, Gilks CF. Molecular typing of multi-drug resistant Shigella dysenteriae type 1 by plasmid analysis and pulsedfield gel electrophoresis. Trans R Soc Trop Med Hyg. 1996;90(6):712-714. https:// doi.org/10.1016/S0035-9203(96)90447-2

7. Foley SL, Lynne AM, Nayak R. Molecular typing methodologies for microbial source tracking and epidemiological investigations of Gram-negative bacterial foodborne pathogens. Infect Genet Evol. 2009;9(4):430-440. https://doi. org/10.1016/j.meegid.2009.03.004

8. Karama M, Gyles CL. Methods for genotyping verotoxin-producing Escherichia coli. Zoonoses Public Health. 2010;57(7-8):447-462. https://doi.org/10.1111/ j.1863-2378.2009.01259.x

9. De R, Ghosh JB, Sen Gupta S, Takeda Y, Nair GB. The role of Vibrio cholerae genotyping in Africa. J Infect Dis. 2013;208 (Suppl 1):32-38. https://doi. org/10.1093/infdis/jit199

10. Goering RV, Kock R, Grundmann $H$, Werner G, Friedrich AW. From theory to practice: Molecular strain typing for the clinical and public health setting. Euro Surveill. 2013;18(4):20383. https://doi.org/10.2807/ese.18.04.20383-en

11. Rahaman MH, Islam T, Colwell RR, Alam M. Molecular tools in understanding the evolution of Vibrio cholerae. Front Microbiol. 2015;6:1040. https://doi. org/10.3389/fmicb.2015.01040 
12. Gilchrist CA, Turner SD, Riley MF, Petri WA, Jr., Hewlett EL. Whole-genome sequencing in outbreak analysis. Clin Microbiol Rev. 2015;28(3):541-563. https:// doi.org/10.1128/CMR.00075-13

13. Leekitcharoenphon P, Nielsen EM, Kaas RS, Lund O, Aarestrup FM. Evaluation of whole genome sequencing for outbreak detection of Salmonella enterica. PLoS One. 2014;9(2):e87991. https://doi.org/10.1371/journal.pone.0087991

14. Jackson BR, Tarr C, Strain E, et al. Implementation of Nationwide Real-time wholegenome sequencing to enhance listeriosis outbreak detection and investigation Clin Infect Dis. 2016;63(3):380-386. https://doi.org/10.1093/cid/ciw242

15. Nadon C, Van Walle I, Gerner-Smidt P, et al. PulseNet International: Vision for the implementation of whole genome sequencing (WGS) for global food-borne disease surveillance. Euro Surveill. 2017;22:pii: 30544. https://doi.org/10.2807/ 1560-7917.ES.2017.22.23.30544.

16. Wachsmuth IK, Kiehlbauch JA, Bopp CA, et al. The use of plasmid profiles and nucleic acid probes in epidemiologic investigations of foodborne, diarrhea diseases. Int J Food Microbiol. 1991;12(1):77-89. https://doi.org/10.1016/0168 1605(91)90049-U

17. Kariuki S, Gilks C, Kimari J, Muyodi J, Waiyaki P, Hart CA. Analysis of Salmonella enterica serotype Typhimurium by phage typing, antimicrobial susceptibility and pulsed-field gel electrophoresis. J Med Microbiol. 1999;48(11):1037-1042. https://doi.org/10.1099/00222615-48-11-1037

18. Gordon MA, Graham SM, Walsh AL, et al. Epidemics of invasive Salmonella enterica serovar enteritidis and $\mathrm{S}$. enterica serovar typhimurium infection associated with multidrug resistance among adults and children in Malawi. Clin Infect Dis. 2008;46(7):963-969. https://doi.org/10.1086/529146

19. Feasey NA, Archer BN, Heyderman RS, et al. Typhoid fever and invasive nontyphoid salmonellosis, Malawi and South Africa. Emerg Infect Dis. 2010;16(9):1448-1451. https://doi.org/10.3201/eid1609.100125

20. Kingsley RA, Msefula CL, Thomson NR, et al. Epidemic multiple drug resistant Salmonella typhimurium causing invasive disease in sub-Saharan Africa have a Salmonella typhimurium causing invasive disease in sub-Saharan Africa have a
distinct genotype. Genome Res. 2009;19(12):2279-2287. https://doi. distinct genotype. Genom
org/10.1101/gr.091017.109

21. Kariuki S, Okoro C, Kiiru J, et al. Ceftriaxone-resistant Salmonella enterica serotype typhimurium sequence type 313 from Kenyan patients is associated with the blaCTX-M-15 gene on a novel IncHI2 plasmid. Antimicrob Agents Chemother 2015;59(6):3133-3139. https://doi.org/10.1128/AAC.00078-15
(1)

22. Leekitcharoenphon $P$, Friis $C$, Zankari $E$, et al. Genomics of an emerging clone of Salmonella serovar typhimurium ST313 from Nigeria and the Democratic Republic of Congo. J Infect Dev Ctries. 2013;7(10):696-706. https://doi.org/10.3855/ jidc. 3328

23. Keddy KH, Sooka A, Musekiwa A, et al. Clinical and microbiological features of Salmonella meningitis in a South African population, 2003-2013. Clin Infect Dis. 2015;61 (Suppl 4):272-282. https://doi.org/10.1093/cid/civ685

24. Okoro CK, Kingsley RA, Quail MA, et al. High-resolution single nucleotide polymorphism analysis distinguishes recrudescence and reinfection in recurrent invasive nontyphoidal Salmonella typhimurium disease. Clin Infect Dis. 2012;54(7):955-963. https://doi.org/10.1093/cid/cir1032

25. Feasey NA, Hadfield J, Keddy KH, et al. Distinct Salmonella enteritidis lineages associated with enterocolitis in high-income settings and invasive disease in lowincome settings. Nat Genet. 2016;48(10):1211-1217. https://doi.org/10.1038/ ng.3644

26. Niehaus AJ, Apalata T, Coovadia YM, Smith AM, Moodley P. An outbreak of foodborne salmonellosis in rural KwaZulu-Natal, South Africa. Foodborne Pathog Dis. 2011;8(6):693-697. https://doi.org/10.1089/fpd.2010.0749

27. Akinyemi KO, Philipp W, Beyer W, Bohm R. Application of phage typing and pulsedfield gel electrophoresis to analyse Salmonella enterica isolates from a suspected outbreak in Lagos, Nigeria. J Infect Dev Ctries. 2010;4(12):828-833. https://doi. org/10.3855/jidc. 744

28. Vaagland H, Blomberg B, Kruger C, Naman N, Jureen R, Langeland N. Nosocomial outbreak of neonatal Salmonella enterica serotype Enteritidis meningitis in a rura hospital in northern Tanzania. BMC Infect Dis. 2004;4:35. https://doi. hospital in northern Tanzanid
org/10.1186/1471-2334-4-35

29. Muvhali M, Smith AM, Rakgantso AM, Keddy KH. Investigation of Salmonella enteritidis outbreaks in South Africa using multi-locus variable-number tandemrepeats analysis, 2013-2015. BMC Infect Dis. 2017;17(1):661. https://doi. repeats analysis, 2013-2015. B
org/10.1186/s12879-017-2751-8

30. Wadula J, Von Gottberg A, Kilner D, et al. Nosocomial outbreak of extendedspectrum beta-lactamase-producing Salmonella isangi in pediatric wards. Pediatr Infect Dis J. 2006;25(9):843-844. https://doi.org/10.1097/01.inf.0000233543. 78070.a2

31. Smith AM, Mthanti MA, Haumann C, et al. Nosocomial outbreak of Salmonella enterica serovar Typhimurium primarily affecting a pediatric ward in South Africa in 2012. J Clin Microbiol. 2014;52(2):627-631. https://doi.org/10.1128/ JCM.02422-13

32. Kariuki S, Revathi G, Muyodi J, et al. Characterization of multidrug-resistant typhoid outbreaks in Kenya. J Clin Microbiol. 2004;42(4):1477-1482. https://doi. org/10.1128/JCM.42.4.1477-1482.2004

33. Keddy KH, Smith AM, Sooka A, Ismail H, Oliver S. Fluoroquinolone-resistant typhoid, South Africa. Emerg Infect Dis. 2010;16(5):879-880. https://doi. org/10.3201/eid1605.091917

34. Lutterloh E, Likaka A, Sejvar J, et al. Multidrug-resistant typhoid fever with neurologic findings on the Malawi-Mozambique border. Clin Infect Dis. 2012;54(8):1100-1106. https://doi.org/10.1093/cid/cis012

35. Neil KP, Sodha SV, Lukwago L, et al. A large outbreak of typhoid fever associated with a high rate of intestinal perforation in Kasese District, Uganda, 2008-2009. Clin Infect Dis. 2012;54(8):1091-1099. https://doi.org/10.1093/cid/cis025
36. Walters MS, Routh J, Mikoleit M, et al. Shifts in geographic distribution and antimicrobial resistance during a prolonged typhoid fever outbreak - Bundibugyo and Kasese Districts, Uganda, 2009-2011. PLoS Negl Trop Dis. 2014;8(3):e2726. and Kasese Districts, Uganda, 2009-2011. PLos

37. Keddy KH, Sooka A, Ismail H, et al. Molecular epidemiological investigation of a typhoid fever outbreak in South Africa, 2005: The relationship to a previous epidemic in 1993. Epidemiol Infect. 2010;139:1239-1245. https://doi.org/ 10.1017/S0950268810002207

38. Tau NP, Smith AM, Wain JR, et al. Development and evaluation of a multiple-locus variable-number tandem-repeats analysis assay for subtyping Salmonella Typhi strains from sub-Saharan Africa. J Med Microbiol. 2017;66(7):937-945. https:// doi.org/10.1099/jmm.0.000526

39. Kariuki S, Revathi G, Kiiru J, et al. Typhoid in Kenya is associated with a dominant multidrug-resistant Salmonella enterica serovar Typhi haplotype that is also widespread in Southeast Asia. J Clin Microbiol. 2010;48(6):2171-2176. https:// doi.org/10.1128/JCM.01983-09

40. Feasey NA, Gaskell K, Wong V, et al. Rapid emergence of multidrug resistant, H58lineage Salmonella typhi in Blantyre, Malawi. PLoS Negl Trop Dis. 2015;9(4):e0003748. https://doi.org/10.1371/journal.pntd.0003748

41. Hendriksen RS, Leekitcharoenphon P, Lukjancenko O, et al. Genomic signature of multidrug-resistant Salmonella enterica serovar typhi isolates related to a massive outbreak in Zambia between 2010 and 2012. J Clin Microbiol. 2015;53(1):262272. https://doi.org/10.1128/JCM.02026-14

42. Wong VK, Holt KE, Okoro $C$, et al. Molecular surveillance identifies multiple transmissions of typhoid in West Africa. PLoS Negl Trop Dis. 2016;10(9):e0004781. https://doi.org/10.1371/journal.pntd.0004781

43. Dalsgaard A, Mortensen HF, Molbak K, Dias F, Serichantalergs O, Echeverria P. Molecular characterization of Vibrio cholerae 01 strains isolated during cholera outbreaks in Guinea-Bissau. J Clin Microbiol. 1996;34(5):1189-1192.

44. Colombo MM, Mastrandrea S, Leite F, et al. Tracking of clinical and environmental Vibrio cholerae $\mathrm{O} 1$ strains by combined analysis of the presence of toxin cassette, plasmid content and ERIC PCR. FEMS Immunol Med Microbiol. 1997;19(1):33-45. https://doi.org/10.1111/j.1574-695X.1997.tb01070.x

45. Aidara A, Koblavi S, Boye CS, et al. Phenotypic and genotypic characterization of Vibrio cholerae isolates from a recent cholera outbreak in Senegal: Comparison with isolates from Guinea-Bissau. Am J Trop Med Hyg. 1998;58(2):163-167. with isolates from Guinea-Bissau. Am J Trop
https://doi.org/10.4269/ajtmh.1998.58.163

46. Dalsgaard A, Forslund A, Sandvang D, Arntzen L, Keddy K. Vibrio cholerae 01 outbreak isolates in Mozambique and South Africa in 1998 are multiple-drug resistant, contain the SXT element and the aadA2 gene located on class 1 integrons. J Antimicrob Chemother. 2001;48(6):827-838. https://doi.org/ integrons. J Antimicrol

47. Folgosa $E$, Mastrandrea S, Cappuccinelli $P$, et al. Molecular identification of pathogenicity genes and ERIC types in Vibrio cholerae $\mathrm{O} 1$ epidemic strains from Mozambique. Epidemiol Infect. 2001;127(1):17-25. https://doi.org/10.1017/ S0950268801005623

48. Ismail H, Smith AM, Sooka A, Keddy KH. Genetic characterization of multidrugresistant, extended-spectrum- beta-lactamase-producing Vibrio cholerae 01 outbreak strains, Mpumalanga, South Africa, 2008. J Clin Microbiol. 2011;49(8):2976-2979. https://doi.org/10.1128/JCM.00293-11

49. Pugliese N, Maimone F, Scrascia M, Materu SF, Pazzani C. SXT-related integrating conjugative element and IncC plasmids in Vibrio cholerae 01 strains in Eastern Africa. J Antimicrob Chemother. 2009;63(3):438-442. https://doi.org/10.1093/ $\mathrm{jac} / \mathrm{dkn} 542$

50. Opintan JA, Newman MJ, Nsiah-Poodoh OA, Okeke IN. Vibrio cholerae 01 from Accra, Ghana carrying a class 2 integron and the SXT element. J Antimicrob Chemother. 2008;62(5):929-933. https://doi.org/10.1093/jac/dkn334

51. Kiiru JN, Saidi SM, Goddeeris BM, Wamae NC, Butaye P, Kariuki SM. Molecular characterisation of Vibrio cholerae O1 strains carrying an SXT/R391-like element from cholera outbreaks in Kenya: 1994-2007. BMC Microbiol. 2009;9:275. https://doi.org/10.1186/1471-2180-9-275.

52. Scrascia M, Maimone F, Mohamud KA, et al. Clonal relationship among Vibrio cholerae $01 \mathrm{El}$ Tor strains causing the largest cholera epidemic in Kenya in the late 1990s. J Clin Microbiol. 2006;44(9):3401-3404. https://doi.org/10.1128/ JCM.00611-06

53. Ngandjio $A$, Tejiokem $M$, Wouafo $M$, et al. Antimicrobial resistance and molecular characterization of Vibrio cholerae 01 during the 2004 and 2005 outbreak of cholera in Cameroon. Foodborne Pathog Dis. 2009;6(1):49-56. https://doi. org/10.1089/fpd.2008.0127

54. Scrascia M, Pugliese N, Maimone $F$, et al. Clonal relationship among Vibrio cholerae O1 El Tor strains isolated in Somalia. Int J Med Microbiol. 2009;299(3):203-207. https://doi.org/10.1016/j.ijmm.2008.07.003

55. Scrascia M, Pugliese N, Maimone F, et al. Cholera in Ethiopia in the 1990s: Epidemiologic patterns, clonal analysis, and antimicrobial resistance. Int J Med Microbiol. 2009;299(5):367-372. https://doi.org/10.1016/j.ijmm.2008.10.004

56. Keddy KH, Nadan S, Govind C, Sturm AW. Evidence for a clonally different origin of the two cholera epidemics of 20012002 and 19801987 in South Africa. J Me Microbiol. 2007;56(Pt 12):1644-1650. https://doi.org/10.1099/jmm.0.47230-0

57. Smith AM, Keddy KH, De Wee L. Characterization of cholera outbreak isolates from Namibia, December 2006 to February 2007. Epidemiol Infect. 2008;136(9):1207-1209. https://doi.org/10.1017/S0950268807009685

58. Ismail H, Smith AM, Tau NP, Sooka A, Keddy KH. Cholera outbreak in South Africa, 2008-2009: Laboratory analysis of Vibrio cholerae O1 strains. J Infect Dis. 2013;208 (Suppl 1):39-45. https://doi.org/10.1093/infdis/jit200

59. Butler D. Cholera tightens grip on Haiti. Nature. 2010;468(7323):483-484. https:// doi.org/10.1038/468483a 
60. Chin CS, Sorenson J, Harris JB, et al. The origin of the Haitian cholera outbreak strain. N Engl J Med. 2011;364(1):33-42. https://doi.org/10.1056/NEJMoa strain. N

61. Talkington D, Bopp C, Tarr C, et al. Characterization of toxigenic Vibrio cholerae from Haiti, 2010-2011. Emerg Infect Dis. 2011;17(11):2122-2129. https://doi. org/10.3201/eid1711.110805

62. Reimer AR, Van DG, Stroika S, et al. Comparative genomics of Vibrio cholerae from Haiti, Asia, and Africa. Emerg Infect Dis. 2011;17(11):2113-2121. https://doi. org/10.3201/eid1711.110794

63. Thompson CC, Freitas FS, Marin MA, Fonseca EL, Okeke IN, Vicente AC. Vibrio cholerae $\mathrm{O} 1$ lineages driving cholera outbreaks during seventh cholera pandemic in Ghana. Infect Genet Evol. 2011;11(8):1951-1956. https://doi.org/10.1016/j. meegid.2011.08.020

64. Mutreja A, Kim DW, Thomson NR, et al. Evidence for several waves of global transmission in the seventh cholera pandemic. Nature. 2011;477(7365):462-465. https://doi.org/10.1038/nature10392

65. Kiiru J, Mutreja A, Mohamed AA, et al. A study on the geophylogeny of clinical and environmental Vibrio cholerae in Kenya. PLoS One. 2013;8(9):e74829. https://doi org/10.1371/journal.pone.0074829

66. Kachwamba Y, Mohammed AA, Lukupulo $\mathrm{H}$, et al. Genetic characterization of Vibrio cholerae 01 isolates from outbreaks between 2011 and 2015 in Tanzania. BMC Infect Dis. 2017;17(1):157. https://doi.org/10.1186/s12879-017-2252-9

67. Kaas RS, Ngandjio A, Nzouankeu A, et al. The Lake Chad Basin, an isolated and persistent reservoir of Vibrio cholerae 01: A genomic insight into the outbreak in Cameroon, 2010. PLoS One. 2016;11(5):e0155691. https://doi.org/10.1371/ journal.pone.0155691

68. Marin MA, Thompson CC, Freitas FS, et al. Cholera outbreaks in Nigeria are associated with multidrug resistant atypical El Tor and non-01/non-0139 Vibrio cholerae. PLoS Negl Trop Dis. 2013;7(2):e2049. https://doi.org/10.1371/journal. pntd.0002049

69. Mercy N, Mohamed AA, Zipporah N, et al. Phenotypic and genetic characterization of Vibrio cholerae 01 isolated from various regions of Kenya between 2007 and 2010. Pan Afr Med J. 2014:19:8. https://doi.org/10.11604/pamj.2014.19.8.2496

70. Mahmud ZH, Islam S, Zaman RU, et al. Phenotypic and genotypic characteristics of Vibrio cholerae $\mathrm{O} 1$ isolated from the Sierra Leone cholera outbreak in 2012. Tran R Soc Trop Med Hyg. 2014;108(11):715-720. https://doi.org/10.1093/trstmh/ tru137

71. Smith AM, Njanpop-Lafourcade BM, Mengel MA, et al. Comparative characterization of Vibrio cholerae $\mathrm{O} 1$ from five sub-Saharan African countries using various phenotypic and genotypic techniques. PLoS One. 2015;10(11):e0142989. https://doi.org/10.1371/journal.pone.0142989

72. Moore S, Miwanda B, Sadji AY, et al. Relationship between distinct African Cholera epidemics revealed via MLVA Haplotyping of 337 Vibrio cholerae isolates. PLoS Negl Trop Dis. 2015;9(6):e0003817. https://doi.org/10.1371/ journal.pntd.0003817

73. Eibach D, Herrera-Leon S, Gil H, et al. Molecular epidemiology and antibiotic susceptibility of Vibrio cholerae associated with a large Cholera outbreak in Ghana in 2014. PLoS Negl Trop Dis. 2016;10(5):e0004751. https://doi org/10.1371/journal.pntd.0004751

74. Pillay DG, Karas JA, Pillay A, Sturm AW. Nosocomial transmission of Shigella dysenteriae type 1. J Hosp Infect. 1997;37(3):199-205. https://doi.org/10.1016/ S0195-6701(97)90248-6

75. Bercion R, Demartin M, Recio C, et al. Molecular epidemiology of multidrug resistant Shigella dysenteriae type 1 causing dysentery outbreaks in Centra African Republic, 2003-2004. Trans R Soc Trop Med Hyg. 2006;100(12):11511158. https://doi.org/10.1016/j.trstmh.2006.02.007

76. Smith AM, Keddy KH, Sooka A, Ismail H, De Jong GM, Hoyland G. Analysis of a temporal cluster of Shigella boydii isolates in Mpumalanga, South Africa, November to December 2007. J Infect Dev Ctries. 2009;3(1):65-70. https://doi. org/10.3855/jidc.107
77. Connor TR, Barker CR, Baker KS, et al. Species-wide whole genome sequencing reveals historical global spread and recent local persistence in Shigella flexneri. Elife. 2015;4:e07335. https://doi.org/10.7554/eLife.07335.:10.

78. Njamkepo E, Fawal N, Tran-Dien A, et al. Global phylogeography and evolutionary history of Shigella dysenteriae type 1. Nat Microbiol. 2016;1:16027. https://doi. org/10.1038/nmicrobiol.2016.27

79. Okeke IN, Ojo O, Lamikanra A, Kaper JB. Etiology of acute diarrhea in adults in southwestern Nigeria. J Clin Microbiol. 2003;41(10):4525-4530. https://doi. org/10.1128/JCM.41.10.4525-4530.2003

80. Tau NP, Meidany P, Smith AM, Sooka A, Keddy KH. Escherichia coli 0104 associated with human diarrhea, South Africa, 2004-2011. Emerg Infect Dis. 2012;18(8):13141317. https://doi.org/10.3201/eid1808.111616

81. Bielaszewska M, Mellmann A, Zhang W, et al. Characterisation of the Escherichia coli strain associated with an outbreak of haemolytic uraemic syndrome in Germany, 2011: A microbiological study. Lancet Infect Dis. 2011;11(9):671-676. https://doi.org/10.1016/S1473-3099(11)70165-7

82. Dallman TJ, Byrne L, Ashton PM, et al. Whole-genome sequencing for national surveillance of Shiga toxin-producing Escherichia coli O157. Clin Infect Dis. 2015;61(3):305-312. https://doi.org/10.1093/cid/civ318

83. Den Bakker HC, Allard MW, Bopp D, et al. Rapid whole-genome sequencing for surveillance of Salmonella enterica serovar enteritidis. Emerg Infect Dis. 2014;20(8):1306-1314. https://doi.org/10.3201/eid2008.131399

84. Moura A, Tourdjman M, Leclercq A, et al. Real-time whole-genome sequencing for surveillance of listeria monocytogenes, France. Emerg Infect Dis. 2017;23(9):14621470. https://doi.org/10.3201/eid2309.170336

85. Zankari E, Hasman H, Kaas RS, et al. Genotyping using whole-genome sequencing is a realistic alternative to surveillance based on phenotypic antimicrobial susceptibility testing. J Antimicrob Chemother. 2013;68(4):771-777. https://doi. org/10.1093/jac/dks496

86. McDermott PF, Tyson GH, Kabera C, et al. Whole-genome sequencing for detecting antimicrobial resistance in nontyphoidal Salmonella. Antimicrob Agents Chemother. 2016;60(9):5515-5520. https://doi.org/10.1128/AAC.01030-16

87. Zhao S, Tyson GH, Chen Y, et al. Whole-genome sequencing analysis accurately predicts antimicrobial resistance phenotypes in Campylobacter spp. Appl Environ Microbiol. 2016;82(2):459-466. https://doi.org/10.1128/AEM.02873-15

88. Stoesser N, Batty EM, Eyre DW, et al. Predicting antimicrobial susceptibilities for Escherichia coli and Klebsiella pneumoniae isolates using whole genomic sequence data. J Antimicrob Chemother. 2013;68(10):2234-2244. https://doi. org $/ 10.1093 / \mathrm{jac} / \mathrm{dkt} 180$

89. Mason A, Foster D, Bradley P, et al. Accuracy of different bioinformatics methods in detecting antibiotic resistance and virulence factors from Staphylococcus aureus whole-genome sequences. J Clin Microbiol. 2018;56(9):e01815-17. https://doi.org/10.1128/JCM.01815-17

90. Koser CU, Ellington MJ, Cartwright EJ, et al. Routine use of microbial whole genome sequencing in diagnostic and public health microbiology. PLoS Pathog. 2012;8(8):e1002824. https://doi.org/10.1371/journal.ppat.1002824

91. Long SW, Williams D, Valson C, et al. A genomic day in the life of a clinical microbiology laboratory. J Clin Microbiol. 2013;51(4):1272-1277. https://doi. org/10.1128/JCM.03237-12

92. Bertelli C, Greub G. Rapid bacterial genome sequencing: Methods and applications in clinical microbiology. Clin Microbiol Infect. 2013;19(9):803-813. https://doi. org/10.1111/1469-0691.12217

93. Allard MW. The future of whole-genome sequencing for public health and the clinic. J Clin Microbiol. 2016;54(8):1946-1948. https://doi.org/10.1128/JCM. 01082-16

94. Oakeson KF, Wagner JM, Mendenhall M, Rohrwasser A, Atkinson-Dunn R. Bioinformatic analyses of whole-genome sequence data in a public health laboratory. Emerg Infect Dis. 2017;23(9):1441-1445. https://doi.org/10.3201/ eid2309.170416 